T. Leng, X. Huang, K.H. Chang, J.C. Chen, X. Zhang and Z. Hu

\title{
A review of graphene radio frequency applications: Now and beyond
}

ABSTRACT. An overview of the recent development in graphene-based radio frequency (RF) applications is presented. For many years, most graphene researchers focused on fundamental physics and properties. High-conductivity graphene ink has been introduced in RF antenna and wireless applications. Effective shielding against electromagnetic (EM) waves provided by graphene-based materials is also introduced as an important application. New lightweight, mechanically flexible, environmentally friendly and low-cost consumable RF devices show us the possibilities for applying graphene in practical applications in everyday life. These pioneering works could lead the way to even more interesting and exciting future applications.

Nanotechnology Perceptions 12 (2016) 153-164

doi: 10.4024/N12LE16R.ntp.12.02 